\title{
Impact of optical phonon scattering on magnetotransport in double-barrier heterostructures
}

\author{
Dae Kwan Kim* and Patrick Roblin ${ }^{\dagger}$ \\ Department of Electrical Engineering, The Ohio State University, Columbus, Ohio 43210 \\ Kwang-Sup Soh ${ }^{\ddagger}$ \\ Department of Physics, Seoul National University, \\ Seoul 151-742, Korea \\ Chul Koo Kim ${ }^{\S}$ \\ Department of Physics and Institute for Mathematical Sciences, Yonsei University, Seoul \\ 120-749, Korea
}

\begin{abstract}
The transport in double-barrier heterostructures of electrons interacting with longitudinal optical phonons in the presence of parallel electric and magnetic fields is analyzed theoretically with the aid of a 3-dimensional quantum transport simulator. Inter Landau state transitions induced by LO-phonon scattering-assisted resonant-tunneling is shown to be an important process with a probability comparable to that of intra Landau state scattering. Anal-
\end{abstract}

\footnotetext{
*E-Mail:kimd@ee.eng.ohio-state.edu

$\dagger$ E-Mail:roblin@ee.eng.ohio-state.edu

${ }^{\ddagger}$ E-Mail:kssoh@phya.snu.ac.kr

§E-Mail:ckkim@phya.yonsei.ac.kr
} 
ysis of the current-voltage characteristics reveals also that the current peak is a periodic function of the inverse of the magnetic field, with a period dependent on the quasi-resonant energy level.

03.65.Sq 72.10.Di 73.40.Gk

Typeset using REVTEX 


\section{INTRODUCTION}

The magnetotransport phenomenal in multiple-barrier heterostructures in which the magnetic is applied parallel to the electric field, was first reported by Mendez, Esaki and Wang 2 . The experimental data acquired in the presence of a magnetic field exhibited abnormal features such as a shift of the onset voltaget, an increase of the peak-to-valley current ratio, as well as the presence of additional shoulders in the valley region of the I-V characteristics 3 . However, these observations have not been fully accounted for on a theoretical basis. This is partly due to the fact that a 3-dimensional treatment of the electron motion is required for rigorously analyzing the impact the quantization in the transverse plane has upon the longitudinal electron motion. Indeed relatively few works analyzing heterostructure devices with a magnetic field parallel to the electric field have been reported until now日.

When the magnetic field and electric field are perpendicular to the interface plane of the composing heterostructure materials, the density of state (DOS) of the electrons in the plane perpendicular to the superlattice direction assumes discrete values corresponding to the Landau levels 6 . When the magnetic field is varied, the DOS of the electrons changes and this strongly impacts the electron motion. The resulting transmission probability and current-voltage characteristics of the heterostructure studied become then quite different from the case with zero magnetic field.

The additional shoulders observed in the valley region of the $\mathrm{I}-\mathrm{V}$ characteristics in the presence of a magnetic field 8 月 8 have been attributed to the longitudinal optical (LO) phonons which is one of the dominant scattering processes of electrons in the III-V semiconductor heterostructure. We present here a model of resonant-tunneling in the presence of of a magnetic field, which includes the electron-LO phonon interaction日, so as to account for the transition of the incident electron to different Landau states. All possible transitions, including intra-Landau state scattering will be considered in our model and numerical analysis. 
The effect of the magnetic field is to quantize the energy levels of the electron into Landau levels. This is due to the fact that the projection of the helicoidal trajectory of the electron on the perpendicular plane corresponds to an harmonic oscillator. To account for the effect of this quantization on the longitudinal motion of electrons in the superlattice direction, we will develop a 3-dimensional transport model. We will then study the abnormal magnetotransport features with the aid of a 3-dimensional quantum simulator which numerically accounts for the contribution of the electron states in the perpendicular plane 1 .

The heterostructure device considered consists of an undoped $\mathrm{Al}_{0.3} \mathrm{Ga}_{0.7} \mathrm{As} / \mathrm{GaAs} /$ $\mathrm{Al}_{0.3} \mathrm{Ga}_{0.7} \mathrm{As}$, double barrier structure sandwiched by heavily doped $n^{+}$-GaAs left and right contact layers. Our analysis only considers tunneling from electrons in the $\Gamma$ valley. The $\Gamma$ conduction-band edge in the absence of an applied voltage for the device studied is shown in the inset of Fig. 1. Spin splitting in the magnetic field is ignored for simplicity. Finally in this paper we are interested in the region of operation for which we have $\hbar w_{c}$ (cyclotron frequency) $\gg k T$, and our numerical calculations will be consequently performed at the low temperature of $T=4.2 \mathrm{~K}$ and for magnetic fields up to 22 Tesla.

In section II, the 3D magnetotransport model used for electrons interacting with longitudinal optical phonons via polor scattering is introduced. The electron state is expanded in the generalized Wannier-Landau basis. Expression for calculating the device transmission coefficient and current-voltage characteristics in the presence of phonon scattering are derived. In section III, the numerical results obtained for the main abnormal magnetotransport features using the above theoretical model are presented and analyzed. Finally in the last section, a brief summary of our results is given.

\section{THE TRANSPORT THEORY}




\section{A. Hamiltonian}

The Hamiltonian of an electron system interacting with longitudinal optical phonons under a magnetic field in the double barrier structure is written as

$$
H=H_{e}+H_{e-p h}
$$

where the free and interaction terms are given by

$$
\begin{aligned}
H_{e} & =\sum_{\lambda} \epsilon(\lambda) c_{\lambda}^{\dagger} c_{\lambda} \\
H_{e-p h} & =\frac{i}{\sqrt{\Omega}} \sum_{\mathbf{q}} \alpha_{\mathbf{q}}\left\{a_{\mathbf{q}} e^{i \mathbf{q} \cdot \mathbf{r}}-a_{\mathbf{q}}^{\dagger} e^{-i \mathbf{q} \cdot \mathbf{r}}\right\}
\end{aligned}
$$

where $c_{\lambda}, c_{\lambda}^{\dagger}\left(a_{\mathbf{q}}, a_{\mathbf{q}}^{\dagger}\right)$ are respectively the destruction and creation operators for electrons (phonons) and $\alpha_{\mathbf{q}}$ is the interaction weight for the phonon wave vector $\mathbf{q}$. Here $\lambda$ stands for the longitudinal crystal wavevector $k_{x}$ and Landau level $L$ and $k_{y}$ in the perpendicular plane, which results from the choice of Landau gauge $\mathbf{6}$ for the vector potential $\mathbf{A}(\mathbf{r})$ and $\mathbf{q}$ is the mode of longitudinal optical phonon. The LO phonons will be represented by an Einstein model with the constant phonon frequency $w$. The Schrödinger equation to be solved is

$$
H|\Psi\rangle=i \hbar \frac{\mathrm{d}}{\mathrm{dt}}|\Psi\rangle
$$

We expand the electron wave function in terms of generalized Wannier functions in the superlattice direction, $x$-direction 10,11 and Landau states in the $(y, z)$ plane,

$$
|\Psi\rangle=\sum_{n, L} \int d k_{y} f\left(k_{y}, L, n\right)\left|k_{y}, L, n\right\rangle
$$

To solve the Schrödinger equation, we calculate the matrix elements of the Hamiltonian in the generalized Wannier-Landau basis. For the free part of Hamiltonian, we find

$$
\left\langle k_{y}, L, n\left|H_{e}\right| k_{y}^{\prime}, L^{\prime}, n^{\prime}\right\rangle=H_{n n^{\prime}}^{e}(L) \delta_{L^{\prime}, L} \delta\left(k_{y}^{\prime}-k_{y}\right),
$$

where

$$
H_{n n^{\prime}}^{e}(L)=H_{n n^{\prime}}+(L+1 / 2) w_{c}(n) \delta_{n n^{\prime}}
$$


with

$$
\begin{aligned}
H_{n n^{\prime}}= & -\frac{\hbar^{2}}{2 a^{2} \sqrt{m^{*}(n) m^{*}(n+1)}} \delta_{n+1, n^{\prime}} \\
& -\frac{\hbar^{2}}{2 a^{2} \sqrt{m^{*}(n) m^{*}(n-1)}} \delta_{n-1, n^{\prime}} \\
& +\left[\frac{\hbar^{2}}{a^{2} m^{*}(n)}+E_{c o n}(n)-e V_{a p p}(n)\right] \delta_{n, n^{\prime}}
\end{aligned}
$$

and $w_{c}(n)=\hbar e B / m^{*}(n)$, the cyclotron frequency at the lattice site $n$. Here, $E_{c o n}(n), V_{a p p}(n)$ are the conduction band edge and applied voltage at the lattice site $n$, respectively. The band model selected consist of a tight-binding band structure in the superlattice direction and a parabolic band structure in the perpendicular plane.

Note that the transversal mass in the Hamiltonian matrix varies in the longitudinal direction. It is convenient to rewrite the Hamiltonian matrix element as

$$
H_{n n^{\prime}}^{e}(L)=\tilde{H}_{n n^{\prime}}(L)+(L+1 / 2) w_{c}(0) \delta_{n n^{\prime}},
$$

in which the position $(n)$ dependent transversal component is now absorbed in

$$
\tilde{H}_{n n^{\prime}}(L)=H_{n n^{\prime}}+(L+1 / 2) \hbar w_{c}(0)\left(\frac{m^{*}(0)}{m^{*}(n)}-1\right) \delta_{n n^{\prime}}
$$

$\tilde{H}_{n n^{\prime}}$ which depends on the perpendicular states L, incorporates the 3-dimensional effects in the longitudinal motion of the electrons.

After some algebra, the matrix element of the interaction Hamiltonian which determines the inter Landau state transitions leads to

$$
\begin{aligned}
\left\langle k_{y}^{\prime}, L^{\prime}, n^{\prime}\left|H_{e-p h}\right| k_{y}, L, n\right\rangle= & \frac{1}{\sqrt{\Omega}} \sum_{q_{x}>0} \frac{1}{q} \sin \left(q_{x} n a\right) \delta_{n^{\prime} n} \\
& \sum_{q_{\perp}}\left\{\alpha_{-} F_{L^{\prime} L}^{k_{y}^{\prime} k_{y}}\left(-q_{\perp}\right) e^{i\left(w t+\phi_{\mathbf{q}}\right)}+\alpha_{+} F_{L^{\prime} L}^{k_{y}^{\prime} k_{y}}\left(+q_{\perp}\right) e^{-i\left(w t+\phi_{\mathbf{q}}\right)}\right\},
\end{aligned}
$$

where $w$ is the phonon frequency, $q_{\perp}$ is the transversal part of $\mathbf{q}$, and $\phi_{\mathbf{q}}$ is the phase of the LO phonon of mode $\mathbf{q}$ (for a more detailed formalism, see ref. [9]). The coefficients $\alpha_{ \pm}$is given by 


$$
\alpha_{-}=\left(2 e F / \epsilon_{0}\right) \sqrt{N_{p h}+1} \text { and } \alpha_{+}=\left(2 e F / \epsilon_{0}\right) \sqrt{N_{p h}}
$$

which corresponds to the emission and absorption of phonons, respectively. Here, $\epsilon_{0}$ is the vacuum dielectric constant, and $N_{p h}$ is the equilibrium number of phonons. $F$ is given as $F=\left(\hbar w_{L O} \epsilon_{0}^{2} / 2\right) \cdot\left(1 / \epsilon_{\text {opt }}-1 / \epsilon_{\text {stat }}\right)$ with phonon energy $\hbar w_{L O}=35.3 \mathrm{meV}$ and $\epsilon_{\text {stat }} / \epsilon_{\text {opt }}=$

1.1664 for GaAs. At liquid Helium temperature, the number of optical phonons $N_{p h}$ is very small, so only the emission of phonons is possible. In Eqn.(10), we introduced the coefficients

$$
\begin{aligned}
F_{L^{\prime} L}^{k_{y}^{\prime} k_{y}}\left( \pm q_{\perp}\right) & =\left\langle k_{y}^{\prime}, L^{\prime}\left|e^{ \pm i q_{\perp} r_{\perp}}\right| k_{y}, L\right\rangle \\
& =\delta\left(k_{y}^{\prime}-k_{y} \pm q_{y}\right) e^{\left[ \pm i q_{z}\left(k_{y}^{\prime}-k_{y} \pm q_{y}\right) l^{2} / 2+i\left(N-N^{\prime}\right)(\theta+\pi / 4)\right]} \bar{F}_{L^{\prime} L}\left(q_{\perp}^{2} l^{2} / 2\right)
\end{aligned}
$$

with

$$
\bar{F}_{L^{\prime} L}\left(\frac{q_{\perp}^{2} l^{2}}{2}\right)=\left(\frac{N^{\prime} !}{N !}\right)^{1 / 2} e^{-q_{\perp}^{2} l^{2} / 4}\left(\frac{q_{\perp}^{2} l^{2}}{2}\right)^{\left(N-N^{\prime}\right) / 2} L_{N^{\prime}}^{N-N^{\prime}}\left(\frac{q_{\perp}^{2} l^{2}}{2}\right)
$$

where $N=\max \left(L, L^{\prime}\right)$ and $N^{\prime}=\min \left(L, L^{\prime}\right)$ are the maximum and the minimum of $L$ and $L^{\prime}$, respectively, $\theta=\operatorname{sign}\left(\mathrm{N}-\mathrm{N}^{\prime}\right) \arctan \left(\mathrm{q}_{\mathrm{y}} / \mathrm{q}_{\mathrm{z}}\right), \mathrm{l}^{2}=\hbar / \mathrm{eB}$, and $L_{N^{\prime}}^{N-N^{\prime}}(x)$ is an associated Laguerre polynomial1 12 .

\section{B. The envelope equation}

Using the obtained matrix elements, Eqn.(5) and (10), in the above subsection, Schrödinger equation can be reduced to the following 3-dimensional envelope equation for $f\left(k_{y}, L, n\right)$,

$$
\begin{aligned}
i \hbar \frac{\mathrm{d}}{\mathrm{dt}} f\left(k_{y}, L, n\right)= & (L+1 / 2) \hbar w_{c 0} f\left(k_{y}, L, n\right)+\sum_{m} \tilde{H}_{n m} f\left(k_{y}, L, m\right) \\
& +\frac{1}{\sqrt{\Omega}} \sum_{L^{\prime} k_{y}^{\prime}}^{q_{x}>0, q_{\perp}} \frac{1}{q}\left[\alpha_{-} \sin \left(q_{x} n a\right) f\left(k_{y}^{\prime}, L^{\prime}, n\right) F_{L L^{\prime}}^{k_{y} k_{y}^{\prime}}\left(-q_{\perp}\right) e^{i\left(w t+\phi_{\mathbf{q}}\right)}\right. \\
& \left.+\alpha_{+} \sin \left(q_{x} n a\right) f\left(k_{y}^{\prime}, L^{\prime}, n\right) F_{L L^{\prime}}^{k_{y} k_{y}^{\prime}}\left(+q_{\perp}\right) e^{-i\left(w t+\phi_{\mathbf{q}}\right)}\right]
\end{aligned}
$$

where $w_{c 0}=e B / m^{*}(0)$, is the cyclotron frequency at the left contact. 
Let us label the quantum state of an incident electron injected at the left contact with the energy $E_{0}=E_{0 x}+\left(L_{0}+1 / 2\right) \hbar w_{c 0}$ using the quantum numbers $\left(E_{0 x}, L_{0}, k_{y 0}\right)$. An electron in the incident ballistic state $\left(E_{0 x}, L_{0}, k_{y 0}\right)$ which is scattered by a phonon will transit to other accessible states $\left(E_{x}, L, k_{y}\right)$ with total energy $E=E_{0} \pm \hbar w_{L O}$ following the emission (-) or absorption $(+)$ of a phonon. Therefore the solution of the envelope Eqn.(I4) is chosen to be of the following form

$$
\begin{aligned}
f\left(k_{y}, L, n\right)= & e^{-i E_{0} t} f_{0}(n) \delta\left(k_{y}-k_{y 0}\right) \delta_{L L_{0}} \\
& +\frac{\alpha_{+}}{\sqrt{\Omega}} e^{-i\left(E_{0}+w\right) t} \sum_{q_{x}>0, q_{\perp}} \frac{1}{q} f_{+}\left(n, q_{\perp}, q_{x}\right) F_{L L_{0}}^{k_{y} k_{y 0}}\left(q_{\perp}\right) e^{-i \phi_{\mathbf{q}}} \\
& +\frac{\alpha_{-}}{\sqrt{\Omega}} e^{-i\left(E_{0}-w\right) t} \sum_{q_{x}>0, q_{\perp}} \frac{1}{q} f_{-}\left(n, q_{\perp}, q_{x}\right) F_{L L_{0}}^{k_{y} k_{y 0}}\left(-q_{\perp}\right) e^{i \phi_{\mathbf{q}}}
\end{aligned}
$$

where $w$ is the phonon frequency $w=w_{L O}, f_{0}$ is the coherent solution for the envelope equation and where $f_{ \pm}$are the scattered waves due to absorption or emission of a phonon, respectively. After substituting this expression into the envelope equation and taking an ensemble average 10 , we arrive at

$$
\begin{aligned}
E_{0 x} f_{0}(n) & =\sum_{m} \tilde{H}_{n m} f_{0}(m)+\sum_{q_{x}>0, m}\left\{H_{S E}^{+}(n, m)+H_{S E}^{-}(n, m)\right\} f_{0}(m), \\
f_{ \pm}\left(n, E_{1 x}, q_{x}\right) & =\sum_{m} G_{n m}\left(E_{1 x}\right) \sin \left(q_{x} m a\right) f_{0}(m),
\end{aligned}
$$

where $H_{S E}^{ \pm}$is the self energy due to absorption and emission of phonons. The self energy is verified to be given by

$$
H_{S E}^{ \pm}(n, m)=\frac{\alpha_{ \pm}^{2}}{\Omega} \sum_{L^{\prime}}^{q_{x}>0, q_{\perp}} \frac{1}{q^{2}} \sin \left(q_{x} n a\right) \sin \left(q_{x} m a\right) G_{n m}\left(E_{1 x}\right)\left[\bar{F}_{L_{0}, L^{\prime}}\left(q_{\perp}^{2} l^{2} / 2\right)\right]^{2}
$$

and $G_{n m}\left(E_{1 x}\right)$ is the longitudinal Green's function in the generalized Wannier basis evaluated using the relation

$$
\sum_{l}\left(E_{1 x}-\tilde{H}\right)_{n l} G_{l m}=\delta_{n m}
$$




\section{Transmission probability and current density}

For an incident electron state $\left(E_{0 x}, L_{0}\right)$ the current entering on the left hand side and leaving the quantum region on the right hand side are respectively given by

$$
\begin{aligned}
& j_{I 0}\left(E_{0 x}, L_{0}\right)=e v_{L}\left(E_{0 x}\right) \\
& j_{T}\left(E_{0 x}, L_{0}\right)=e\left|f_{0}\left(n_{R}\right)\right|^{2} v_{R}\left(E_{0 x}\right)+j_{ \pm, p h}\left(E_{0 x}, L_{0}\right) .
\end{aligned}
$$

Here the phonon-assisted current component $J_{ \pm, p h}$ is given by

$$
j_{ \pm, p h}\left(E_{0 x}, L_{0}\right)=e \frac{\alpha_{ \pm}^{2}}{\Omega} \sum_{L^{\prime}}^{q_{x}>0, q_{\perp}} \frac{1}{q^{2}}\left|f_{ \pm}\left(n_{R}, q_{\perp}, q_{x}\right)\right|^{2}\left[\bar{F}_{L_{0}, L^{\prime}}\left(q_{\perp}^{2} l^{2} / 2\right)\right]^{2} .
$$

In the above equations, $v_{L}$ and $v_{R}$ are the electron velocity at the left and right contact, and $n_{R}$ is the lattice-site index of the right contact. The transmission probability from the left to right contact is then defined as

$$
T_{L \rightarrow R}\left(E_{0 x}, L_{0}\right)=\frac{\left[j_{T 0}\left(E_{0 x}, L_{0}\right)+j_{+, p h}\left(E_{0 x}, L_{0}\right)+j_{-, p h}\left(E_{0 x}, L_{0}\right)\right]}{j_{I 0}\left(E_{0 x}, L_{0}\right)}
$$

Before calculating the current, the electron chemical potential $\mu(B)$ at the left contact corresponding to the doping density $n$ in presence of the magnetic field $B$, should be determined. As is shown in Fig. 1, the chemical potential is found to exhibit an oscillatory behavior in $1 / B$ as the magnetic field varies. The chemical potential $\mu(B)$ in the left contact is obtained from the carrier density through the following integration relation,

$$
n=N_{D}=\left(\sqrt{2 m^{*}} / \pi \hbar\right) D(B) \sum_{L_{0}=0}^{\infty} \int_{0}^{\infty} \frac{f_{F D}\left(E_{0 x}, \mu_{e f f}\left(B, L_{0}\right)\right)}{\sqrt{E_{0 x}}} d E_{0 x},
$$

where $D=e B / h$ is a degeneracy factor in the plane perpendicular to the superlattice direction and where the Fermi-Dirac function $f_{F D}$ is given by

$$
f_{F D}\left(E_{0 x}, \mu_{e f f}\left(B, L_{0}\right)\right)=\frac{1}{\exp \left[\left(E_{0 x}-\mu_{e f f}\left(B, L_{0}\right)\right) / k T\right]+1} .
$$

Here we have found it convenient to introduce the effective chemical potential $\mu_{e f f}$ for the Landau sub-band $L_{0}$ which is related to the actual electron chemical potential $\mu(B)$ according to 


$$
\mu_{e f f}\left(B, L_{0}\right)=\mu(B)-\left(L_{0}+1 / 2\right) \hbar w_{c 0}(B) .
$$

$\mu_{e f f}$ can be interpreted as the effective chemical potential of the electrons in the Landau state $L_{0}$ in the presence of a magnetic field. Thus, the total current density is

$$
I_{\text {tot }}=(e D(B) / \pi \hbar) \sum_{L_{0}=0}^{\infty} \int_{0}^{\infty} T_{L \rightarrow R}\left(E_{0 x}, L_{0}\right) f_{F D}\left(E_{0 x}, \mu_{e f f}\left(B, L_{0}\right)\right) d E_{0 x}-[L \leftrightarrow R],
$$

where the second term in the right-hand side is the backward contribution to the total current. At very low temperatures such as $4.2 \mathrm{~K}$, the current for a occupied sub-band $L_{0}$ reduces to

$$
I_{t o t, L_{0}} \approx(e D / \pi \hbar) \int_{0}^{\mu_{e f f}} d E_{0 x} T_{L \rightarrow R}\left(E_{0 x}, L_{0}\right)-[L \leftrightarrow R]
$$

Therefore, the area from 0 to $\mu_{\text {eff }}$ of the transmission probability determines the contribution of each occupied sub-band to the total current.

\section{NUMERICAL RESULTS}

As mentioned above the heterostructure device considered consists of an undoped $\mathrm{Al}_{0.3} \mathrm{Ga}_{0.7} \mathrm{As} / \mathrm{GaAs} / \mathrm{Al}_{0.3} \mathrm{Ga}_{0.7} \mathrm{As}$, double barrier structure (see inset of Fig. 四) sandwiched by heavily doped $n^{+}$-GaAs left and right contact layers. The barriers and well are both $50 \AA$ wide. The donor density in the contact regions is $1 \times 10^{18} / \mathrm{cm}^{3}$. The donors are assumed to be completely ionized due to the heavy doping density. The value of the electron effective mass used for GaAs and $\mathrm{Al}_{0.3} \mathrm{Ga}_{0.7} \mathrm{As}$ is $m^{*}=0.067 m_{0}$ and $0.092 m_{0}$, respectively.

The numerical calculation starts with the calculation of the matrix element $\bar{F}_{L^{\prime} L}\left(q_{\perp}^{2} l^{2} / 2\right)$ which is required to evaluate the self energy $H_{S E}^{ \pm}(n, m)$ associated with the inter Landau state transitions for all possible values of $\left(E_{0 x}, L_{0}\right)$. This self energy is then used to solve the envelope equation, and obtain the transmission coefficients. The current density is finally obtained by integrating the transmission coefficient obtained over the incident energy. We confined our numerical analysis to a single-sequential phonon scattering event since this is a quite reasonable approximation for the small heterostructure device under investigation. 
In order to calculate the currents, it is required to calculate the chemical potential $\mu$ as a function of the applied magnetic field $B$. Using Eqn.(23) the chemical potential $\mu(B)$ calculated for several temperatures is plotted in Fig. 目 versus $B$. The chemical potential $\mu(B)$ is found to be a periodic function in $1 / B$. This figure clearly indicates that in the region of strong magnetic fields $(B>10$ Telsa) using the approximation of a constant Fermi energy $E_{f}$ would introduce a rather large error in the chemical potential. This would in turn adversely affect the accuracy of the device current calculated as is verified in Fig. 囵. However at the temperature of $100 K$, the oscillating behavior in $\mu$ disappears completely due to thermal population of all the Landau states near the Fermi energy.

In Fig. Q, the transmission probability in a flat band (no applied voltage) is plotted for two magnetic fields of $B=10$ and 20 Telsa at $T=4.2 \mathrm{~K}$. At this low temperature, the emission of phonons dominates since the number of phonons in the system is negligible. The peaks beyond the main peak centered at $94 \mathrm{meV}$, are phonon-assisted resonant tunneling peaks which satisfy the following energy conservation relation

$$
E_{0 x}-\hbar w=E_{x}+\Delta L \cdot \hbar w_{c 0}
$$

where $\left(E_{0 x}, L_{0}\right)$ is the incident electron state and $\left(E_{x}, L\right)$ is the scattered state after the emission of one phonon and with $\Delta L=\left(L-L_{0}\right)$. The energy of the phonon is chosen to be $\hbar w=35.3 \mathrm{meV}$ for GaAs contact layers for simplicity. A single-sequential phononemission has been assumed. This was reported to be a valid approximation in a small size system like the double barrier structure considered 3 阳 The peak indicated by $\Delta L=0$ in Fig. 2 arises when an incident electron moves in the structure with the emission of a single phonon and the Landau level remains conserved while the peaks $\Delta L=1$ and 2 indicate that phonon-assisted resonant-tunneling induced a transition to higher Landau levels. As the magnetic field is increased, the spacing $\hbar w_{c}(B)$ between $\Delta L$ neighboring peaks increases in proportion to the applied external magnetic field where as the spacing between the main peak and $\Delta L=0$ peak remains constant as it is set by the phonon energy $\hbar w$. For an incident state with Landau levels $L_{0}>0$, transitions of the type $\Delta L=-n$ arise (with $n$ 
an integer verifying $n \leq L_{0}$ ), and additional peaks appears between the main and $\Delta L=0$ peaks.

The matrix element $\bar{F}_{L^{\prime} L}\left(q_{\perp}^{2} l^{2} / 2\right)$ between Landau states in Eqn.(13) determines the strength of the inter Landau state transitions in the transmission coefficient. For example, at $B=10$ Telsa its argument $q_{\perp}^{2} l^{2} / 2$ assume values in the range $(0.025,252)$ depending on the phonon mode. After the matrix $\bar{F}_{L^{\prime} L}\left(q_{\perp}^{2} l^{2} / 2\right)$ is summed over all possible phonon modes $q_{\perp}$, the inter Landau state transition probability is obtained. The analysis of the various scattering-assisted resonant-tunneling processes induced by LO phonon emission, indicates that the transmission probabilities for scattering events involving the transition between two different Landau states $(\Delta L>0)$ is of the same order as the transmission probability for intra Landau state $(\Delta L=0)$ scattering events. Fig. 2 clearly shows this property.

Fig. 3, 田, and 5 shows the electron current density calculated under various conditions. The current density is obtained from the integration over the incident electron energy of the transmission coefficient $T\left(E_{0 x}, L_{0}\right)$ multiplied by the Fermi-Dirac occupation function which is almost a step function at the low temperature considered. A broadening of the Landau levels up to $2 \mathrm{meV}$ was considered, but there were no noticeable difference in our results for the calculated currents for $B>5$ Telsa since we have $\hbar w_{c 0}(B=5)=8.6 \mathrm{meV} \gg 2 \mathrm{meV}$. We observe in Fig. 3 and 5 , the presence of small additional shoulders of almost equal width in the valley region of the current-voltage curve. These shoulders originate from that fact that the phonon-assisted resonant-tunneling peaks in the transmission coefficient are spaced by $\hbar w_{c}$ above the main quasi-resonant energy peak $E_{r}$.

Before discussing the impact of phonon scattering, let us first briefly describe the main effects of the magnetic field upon the current of the resonant tunneling diode (RTD) in the absence of phonon scattering. As can be seen in Fig. 通 and the magnetic field is found to induce a shift of the onset voltage of the RTD I-V characteristic by the amount $\Delta V_{D, o n} / 2=[\mu(0)-\mu(B)]+\hbar w_{c 0}(B) / 2$, and to enhance the value of peak current of the RTD. Fig. 国reveals another interesting feature regarding the dependence of the RTD currentvoltage characteristics upon the magnetic field. In this Figure the diode current is measured 
at $V_{D}=0.146$ Volt which is in the current peak region of the RTD I-V curve. Note that phonon scattering was not included in this calculation as coherent (ballistic) tunneling gives the dominant contribution to the peak current. The RTD current measured at that fixed diode voltage is seen to exhibits an oscillatory behavior versus $1 / B$ which increases

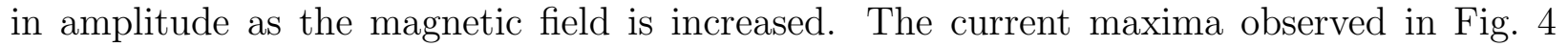
for the current versus $B$ are found to occur for values $B_{n}$ of the magnetic field satisfying the following relation $\mu\left(B_{n}\right)-(n+1 / 2) \hbar w_{c 0}\left(B_{n}\right)=E_{r}\left(V_{D}\right)$ where the quasi-resonant level $E_{r}\left(V_{D}\right) \simeq E_{r}(0)-V_{D} / 2$ is varying with the diode voltage. This leads to the interesting result that its periodicity versus $1 / B$ which is approximately $\hbar e / m^{*}(0)\left[\mu(0)-E_{r}\left(V_{D}\right)\right]^{-1}$, depends on the quasi-resonant energy level $E_{r}\left(V_{D}\right)$; for a smaller value of $E_{r}$, one obtains an increased oscillatory behavior in the current-magnetic field curve.

As can be seen Fig. 3 and 5, the effect of phonon scattering on the RTD current density is to shift the current-voltage characteristic downward compared to the one obtained in the absence of scattering. Another effect is to increase the current and to introduce additional shoulders in the valley region of the current-voltage characteristic. The downward shift of onset voltage results from the negative value of the real part of the self energy induced by the scattering of the electron by the phonons. For magnetic fields of $B=10$ and 20 Telsa, the downward shifts obtained in the current-voltage characteristic are found to be 3.6 and $4.0 \mathrm{mV}$, respectively. The small shoulders at $B=20$ Telsa in the current valley region of Fig. 5 become larger than for the case of $B=10$ Telsa. Thus in stronger magnetic fields, these additional shoulders in the valley region become higher and their intervals becomes wider, which agrees with the reported experiments 3 :

To account for the origin of the shoulder at $V_{D}=0.187$ Volt, let us consider the inset of Fig. 5. At $B=20$ Telsa, only the two lowest Landau levels $L_{0}=0$ and 1 contribute to the diode current. The effective chemical potentials defined in Eqn.(25) for the $L_{0}=0$ and 1 states are respectively $\mu_{\text {eff }}=37.3$ and $2.8 \mathrm{meV}$. They are indicated by two arrows in the inset of Fig. 5. At $V_{D}=0.187$ Volt, both the peaks $\Delta L=0$ and -1 of transmission coefficients for the $L_{0}=0$ and 1 incident states are respectively included into the integration 
range of the current Eqn.(27), explaining the formation of the shoulders.

Currently, it is difficult to compare our theoretical results in a quantitative way to the experiments reported in Ref. 2 and 3. The reasons are as follows. First, the authors of Ref. 2 concluded that additional experiments are needed to confirm the weak minima of their preliminary data in the valley current region for strong magnetic fields. Second, our test device doesn't have a spacer layer in the emitter region, while the device structure of Ref. 3 have a spacer layer where a discrete triangular well state is formed. The shoulders therein corresponding to $\delta L \neq 0$ beyond the main peak was reported to be as high as almost $2 / 5$ of the main peak for extremely strong magnetic field, which were clarified to originate from the assistance of LO phonons to the electron current. Our results together with Ref. 2 and 3 lead to the conclusion that the spacer layer tends to enhance the strength of the shoulders beyond the main peak. But in order to explain such high shoulders, other contributions due to scatterings of electrons by interface roughness, acoustic phonon, etc., might also be needed.

\section{SUMMARY}

In our paper we have investigated the transport of electrons interacting with LO phonons in a double-barrier heterostructure with a magnetic field applied parallel to the electric field. The effect of the magnetic field is to quantize the energy of the electron in the perpendicular plane to the magnetic field. To account for the effects of a magnetic field applied in parallel with the superlattice direction, a 3-dimensional transport model was developed which accounts for the variation of the transverse mass and the associated variation of the cyclotron frequency across the heterostructure.

In order to determine the inter Landau state transitions in the transmission probability,

all the matrices $\bar{F}_{L^{\prime} L}\left(q_{\perp}^{2} l^{2} / 2\right)$ for allowed phonon modes have been calculated numerically. It was established that the inter Landau state transitions due to LO phonon scattering are as probable as intra Landau state scattering. 
For a fixed diode voltage in the peak region of the RTD current-voltage curve, the RTD current versus the magnetic field was found to be a periodic function of $1 / B$. The current maxima have an $1 / B$ period which is approximately given by $\hbar e / m^{*}(0) /\left(\mu(0)-E_{r}\left(V_{D}\right)\right)$. The dependence of this $1 / B$ period on the quasi-resonant energy level $E_{r}\left(V_{D}\right)$ of our test double barrier structure indicates that magnetotransport can be used for the spectroscopic analysis of RTDs for diode voltages in the peak current region.

A theory and associated numerical analysis using scattering matrix elements was presented to describe the impact of electron-LO phonon interaction in the I-V curve in the presence of both parallel electric and magnetic fields. The results obtained were found to be in qualitative agreement with the experimental results reported by Ref. 2 and 3. However

for further understanding and improved quantitative fit of the weak shoulders in the valley region of the double barrier IV, more theoretical work on magnetotransport including scattering processes such as interface roughness and acoustic phonon scattering, etc., is required in addition to LO phonon scattering studied in this paper.

\section{Acknowledgements}

This work was supported in part by the Korea Science and Engineering Foundation (KOSEF). The first author is grateful to the EE department of the Ohio-State University for hosting him during the course of this research. The authors would like to thank the anonymous reviewers for their suggestions. 


\section{REFERENCES}

${ }^{1}$ B. K. Ridley, Quantum Processes in Semiconductors (Oxford University Press, Oxford, 1999).

${ }^{2}$ E. E. Mendez, L. Esaki, and W. I. Wang, Phys. Rev. B 33, 2893 (1986).

${ }^{3}$ M. L. Leadbeater, E. S. Alves, L. Eaves, M. Henini, O. H. Hughes, A. Celeste, J. C. Portal, G. Hill, and M. A. Pate, Phys. Rev. B 39, 3438 (1989).

${ }^{4}$ L. Canali, M. Lazzarino, L. Sorba, and F. Beltram, Phys. Rev. Lett. 76, 3618 (1996).

${ }^{5}$ K. S. Chan, F. W. Sheard, G. A. Toombs, and L. Eaves Phys. Rev. B 56, 1447 (1997).

${ }^{6}$ L. D. Landau and E. M. Lifshitz, Quantum Mechanics: Non Relativistic Theory (Pergamon Press, Oxford, 1965).

${ }^{7}$ H. Asahi, M. Tewordt, R. T. Syme, M. J. Kelly, V. J. Law, D. R. Mace, J. E. F. Frost, D. A. Ritchie, G. A. C. Jones, and M. Pepper, Appl. Phys. Lett. 59, 803 (1991).

${ }^{8}$ For certain devices with p-type $\mathrm{Si} / \mathrm{SiGe}$ double barrier structures which has quite different band structures from our case, the shoulders beyond a main peak can be induced due to non-parabolic, light and heavy hole bands even in the absence of other scattering events. See, for example, A. Zaslavsky, D. A. Grützmacher, S. Y. Lin, T. P. Smith III, R. A. Kiehl, and T. O. Sedgwick, Phys. Rev. B 47, 16036 (1993); D.-Y. Lin, C.-W. Chen, and G. Y. Wu, Phys. Rev. B 57, 4599 (1997).

${ }^{9}$ N. S. Wingreen, K. W. Jacobsen, and J. W. Wilkins, Phys. Rev. Lett., 61, 1396 (1988).

${ }^{10}$ P. Roblin and W.-R. Liou, Phys. Rev. B 47, 2146 (1993); P. Sotirelis and P. Roblin, Phys. Rev. B 51, 13381 (1995).

${ }^{11}$ W. Kohn and J. R. Onffroy, Phys. Rev. B 8, 2485 (1973); J. G. Gay and J. R. Smith, Phys. Rev. B 11, 4906 (1975).

12 D. Pfannkuche and R. R. Gerhardts, Phys. Rev. B 46, 12606 (1992). 


\section{FIGURES}

FIG. 1. The chemical potentials at $n^{+}=1 \times 10^{18} / \mathrm{cm}^{3}$ as a function of the magnetic field for various temperatures. The solid, dotted, dashed, dot-dashed, double dot-dashed lines correspond

to $T=4.2,10,20,40$, and $100 \mathrm{~K}$, respectively. The bold dotted line is the Fermi energy $E_{f}$ at $T=0, B=0$. The inset figure is the conduction band profile of the resonant-tunneling structure.

FIG. 2. Transmission probability for the incident Landau state $L_{0}=0$ with no applied voltage at $T=4.2 K$. The dotted and solid lines correspond to $B=10$ and 20 Telsa, respectively.

FIG. 3. RTD current densities versus applied voltage for $B=10$ Telsa at $T=4.2 K$. The dotted, solid lines correspond to the RTD currents calculated without and with LO phonon scattering respectively, and the dashed line is for the RTD current in the absence of both an applied magnetic field and phonon scattering. In inset a plot showing the $I-V$ characteristic in the absence of both an applied magnetic field and scattering for a wider diode voltage range is also given as a reference.

FIG. 4. Current density (solid line) plotted versus the magnetic field for an applied voltage of 0.146 Volt. The dotted line corresponds to the RTD current density calculated in the approximation of a constant Fermi energy $(\mu(B)=\mu(0))$. The dashed line in the center corresponds to the current value in the absence of a magnetic field $(B=0)$.

FIG. 5. RTD current densities versus applied voltage for $B=20$ Telsa at $T=4.2 K$. The dotted, solid lines correspond to the RTD currents calculated without and with LO phonon scattering, respectively. The dashed line is for the RTD current in magnetic free case without scatterings. The inset shows the transmission probability for the two lowest Landau states $L_{0}=0$ and 1 at the voltage $V_{D}=0.187 \mathrm{~V}$. 


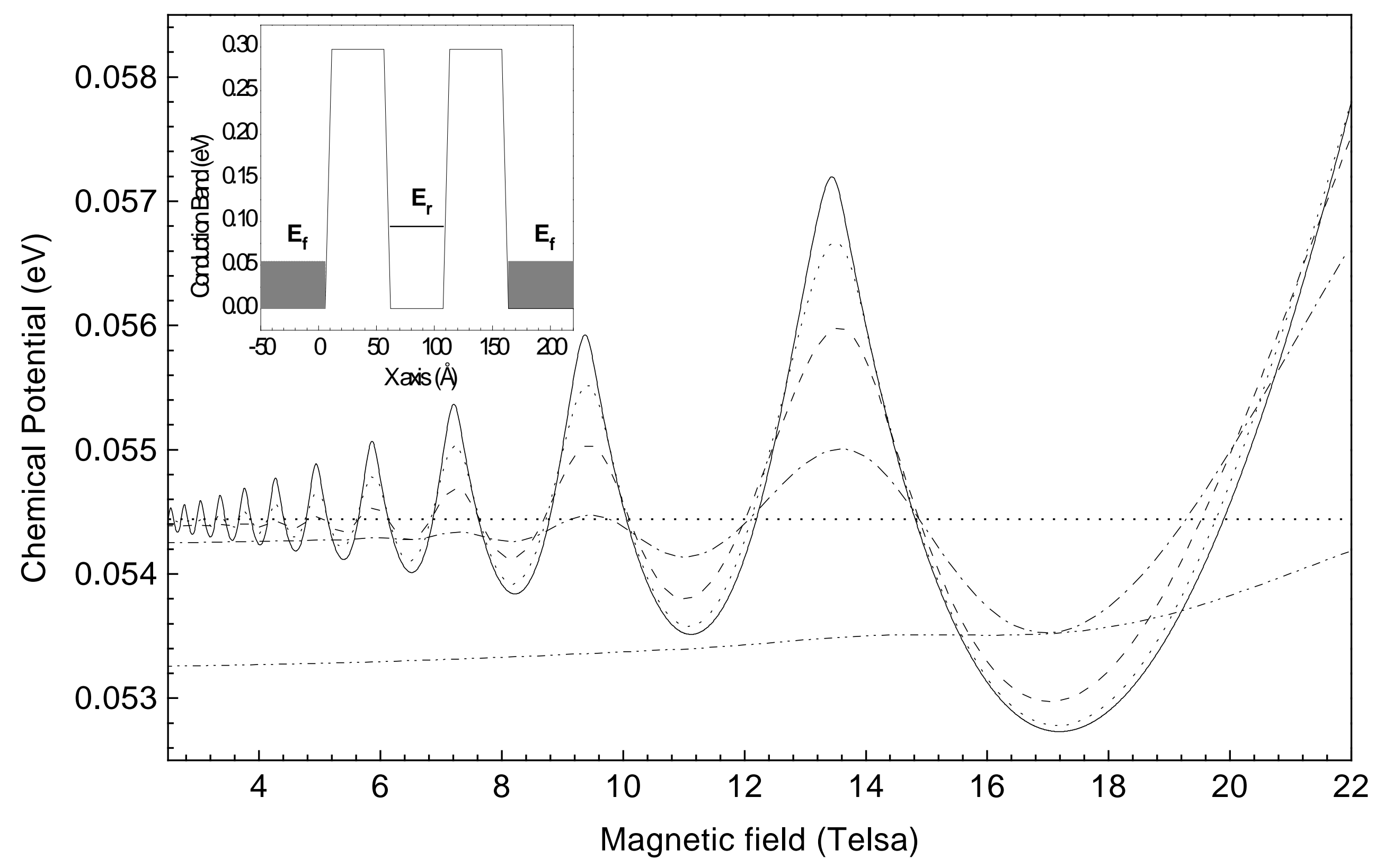




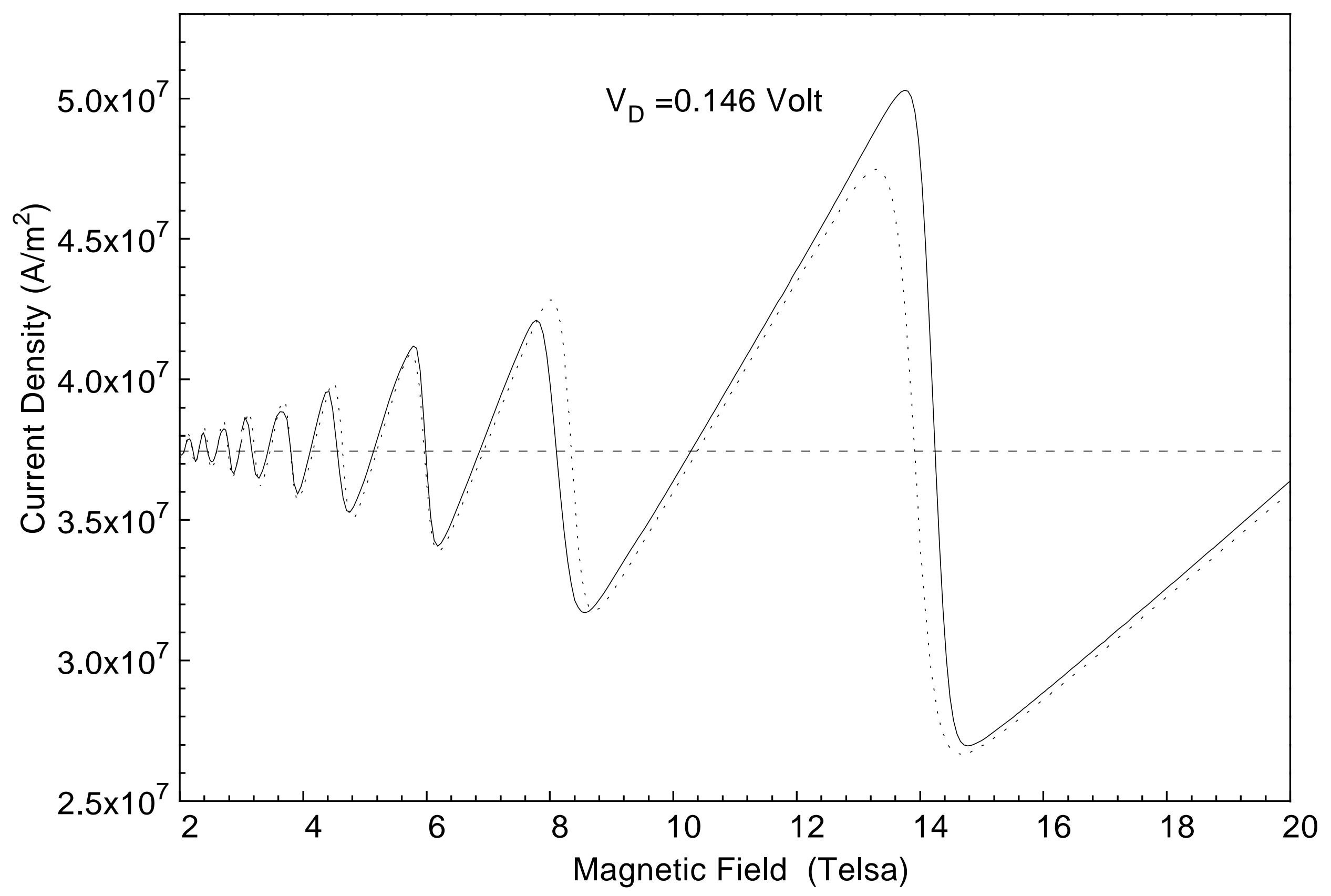




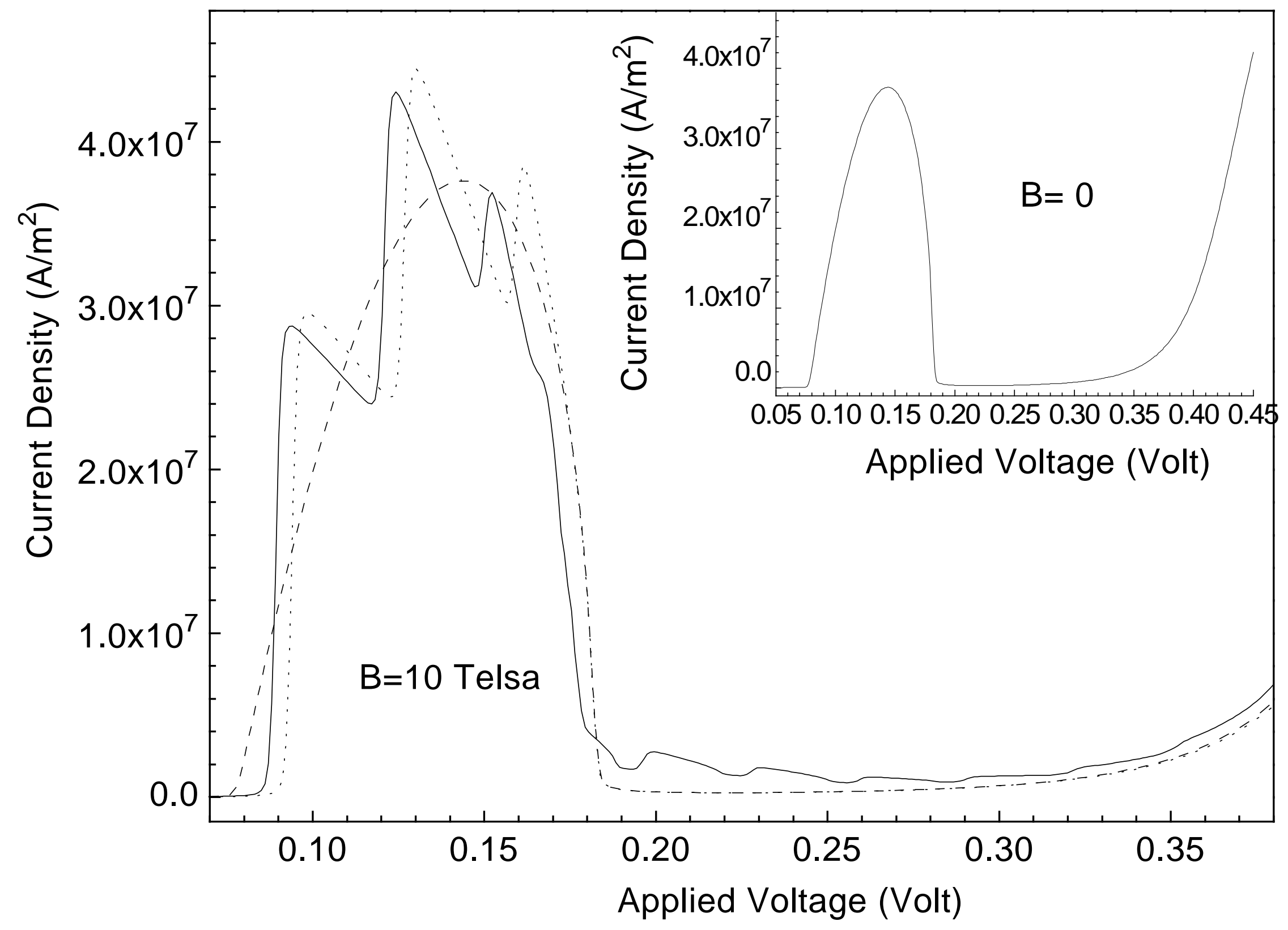




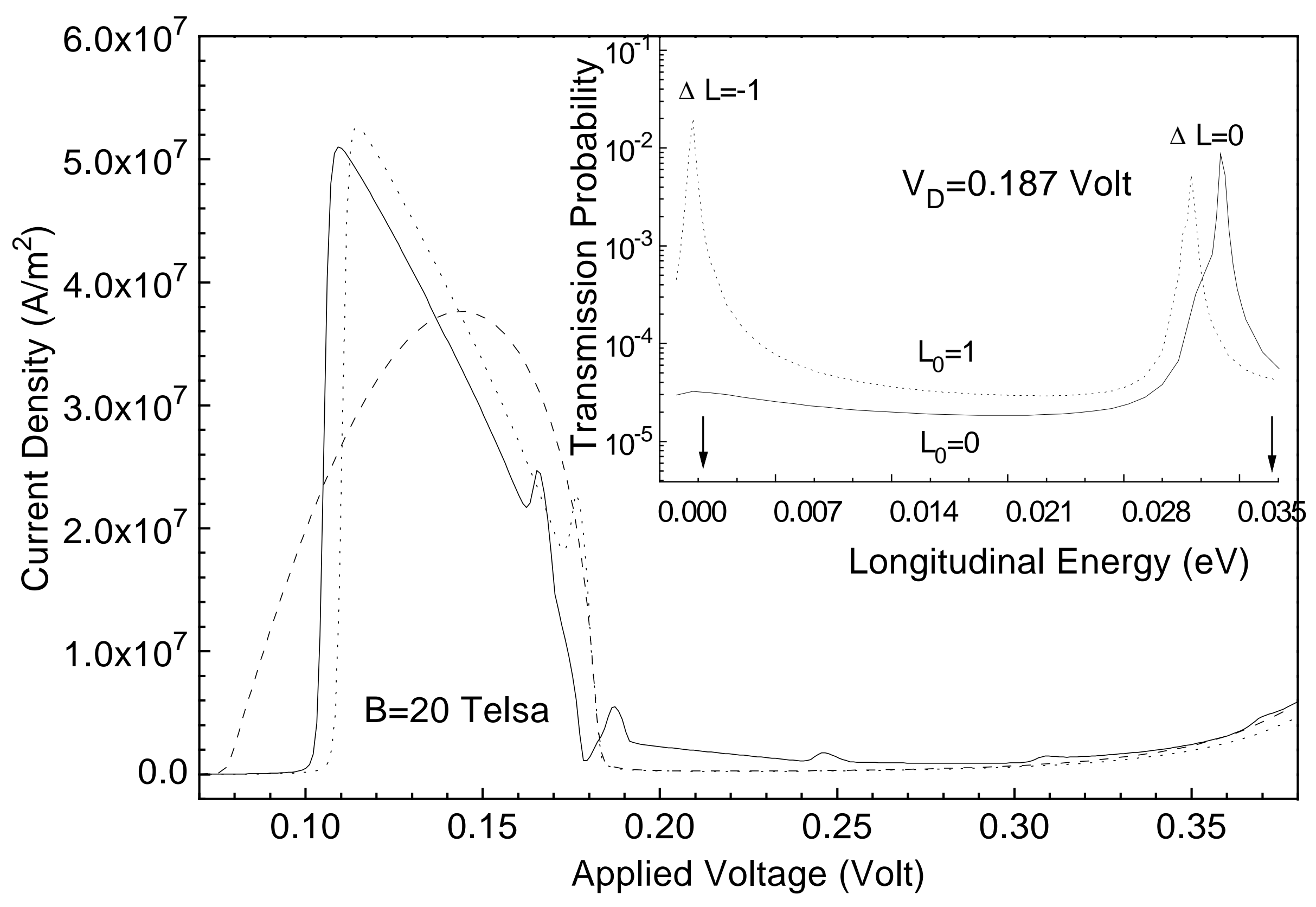




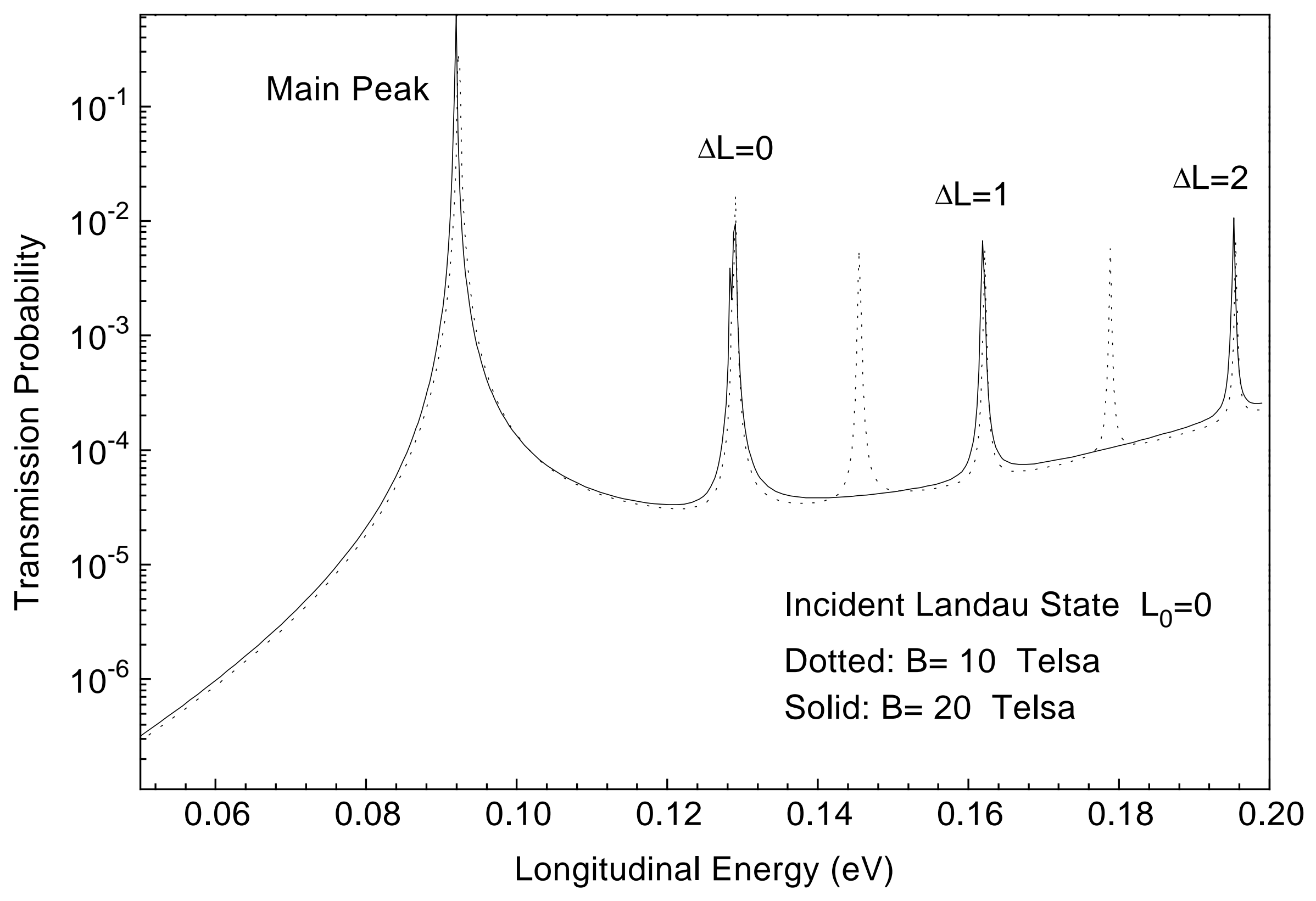

